\title{
A Rapid Gene-Based Genome-Wide Association Test with Multivariate Traits
}

\author{
Saonli Basu ${ }^{a}$ Yiwei Zhang ${ }^{a}$ Debashree Ray ${ }^{a} \quad$ Michael B. Miller ${ }^{b}$ \\ William G. lacono ${ }^{b}$ Matt McGue ${ }^{b}$ \\ a Division of Biostatistics, School of Public Health, and ${ }^{\mathrm{b}}$ Department of Psychology, University of Minnesota, \\ Minneapolis, Minn., USA
}

\section{Key Words}

Multivariate regression . Gene-based genome-wide association studies . Multivariate trait

\begin{abstract}
Objectives: A gene-based genome-wide association study (GWAS) provides a powerful alternative to the traditional single single nucleotide polymorphism (SNP) association analysis due to its substantial reduction in the multiple testing burden and possible gain in power due to modeling multiple SNPs within a gene. A gene-based association analysis on multivariate traits is often of interest, but it imposes substantial analytical as well as computational challenges to implement it at a genome-wide level. Methods: We propose a rapid implementation of the multivariate multiple linear regression (RMMLR) approach in unrelated individuals as well as in families. Our approach allows for covariates. Moreover, the asymptotic distribution of the test statistic is not heavily influenced by the linkage disequilibrium (LD) among the SNPs and hence can be used efficiently to perform a gene-based GWAS. We have developed a corresponding $\mathrm{R}$ package to implement such multivariate gene-based GWAS with this RMMLR approach. Results: Through extensive simulation, we compared several approaches for both single and multivariate traits. Our RMMLR approach maintained a correct
\end{abstract}

type I error level even for sets of SNPs in strong LD. It also demonstrated a substantial gain in power to detect a gene when it is associated with a subset of the traits. We also studied performances of the approaches on the Minnesota Center for Twin Family Research dataset. Conclusions: In our overall comparison, our RMMLR approach provides an efficient and powerful tool to perform a gene-based GWAS with single or multivariate traits and maintains the type I error appropriately.

(c) 2013 S. Karger AG, Basel

\section{Introduction}

The rapid progress in genotyping technology has greatly facilitated our understanding of the genetic aspect of various diseases. Several genome-wide association studies (GWAS) have been published on various complex diseases, where genotype data on a large number of single nucleotide polymorphisms (SNPs) are collected to study the association between these SNPs and a disease [1]. Currently, most existing statistical methods for detecting genetic association focus on analyzing SNPs one-byone [2].

Although, new SNPs are found to be associated with these diseases, little of the genetic risk or heritability for 
these diseases is explained by the additive effects of these SNPs. Studying one SNP at a time may not be sufficient to understand complex diseases. Analyses focusing on individual SNPs may have several limitations: (a) a large number of comparisons increases the probability of false positives and a stringent correction could lead to loss of power and (b) each SNP alone may have very little effect on the risk of disease [3], but multiple causal variants together may increase the risk substantially. A single SNP analysis may not be optimal here; especially in the presence of multi-SNP effects.

Hence, a gene-based association analysis is often of interest in GWAS. This approach substantially reduces the burden of multiple testing at a GWAS scale. Moreover, by pooling information on multiple SNPs within a gene, one could gain substantial power in detecting association. Current gene-based association approaches are mostly limited to combining single SNP association $p$ values to propose a gene-based test statistic [4-6]. One limitation of these gene-based statistics is that combining single SNP $p$ values ignores the potential joint effect of multiple SNPs within a gene. Joint modeling of SNPs within a gene could have a substantial gain in power over these existing gene-based tests. Moreover, the current approaches typically require permutation or simulation testing to account for the correlation between the SNPs as well as the gene size. VEGAS [7] and the PLINK setbased test [8] are 2 such available approaches for performing gene-based tests. They can be computationally very intensive to perform a gene-based GWAS, since they require a large number of permutations to correct for multiple testing. Hence their usefulness to conduct a gene-based GWAS is somewhat limited. Moreover, these approaches have been implemented for univariate phenotypes.

In the study of a complex disease, several correlated traits are often measured as risk factors for the disease. For example in the study of thrombosis, the intermediate correlated phenotypes, such as Factor VII, VIII, IX, XI, XII, and von Willebrand factor, jointly predict the risk of developing thrombosis $[9,10]$. There may be genetic variants affecting several of these traits. Analyzing multiple disease-related phenotypes could potentially increase power to detect association of SNPs/genes with a disease. Moreover, this joint analysis could reveal some pleiotropic genes involved in the biological development of the disease. It has been shown that modeling multivariate phenotypes may increase the power over analyzing individual phenotypes separately in genetic association studies $[11,12]$.
Few approaches have been developed to perform association analysis with multivariate traits at a GWAS level. O'Reilly et al. [13] proposed MultiPhen to detect association between multivariate traits and a SNP with unrelated individuals. It uses ordinal regression to regress a SNP on a collection of phenotypes and tests whether all regression parameters corresponding to the phenotypes in the model are significantly different from zero. van der Sluis et al. [14] proposed a method - TATES - for testing association between multiple traits and multiple SNPs using an extended Simes procedure on the univariate $\mathrm{p}$ values derived from a univariate trait and single SNP association analysis. Recently, Maity et al. [15] have proposed a kernel machine method for joint analysis of multiple genetic variants, which treats the SNP effects as a random effect in the model. Their test for association between multiple SNPs and the phenotypes is equivalent to testing the variance components corresponding to the random effect in a multivariate linear mixed model. Implementation of this approach requires parametric bootstrapping to estimate the distribution of the test statistic and could be computationally intensive at a GWAS level. Additionally, this method has been proposed for unrelated individuals.

Recently, the advantage of using canonical correlation analysis (CCA) to perform gene-based tests on multivariate phenotypes has been elaborately discussed in Tang and Ferreira [16]. Previously, Ferreira and Purcell [17] proposed a multivariate test of association based on CCA to simultaneously test the association between a single SNP and multiple phenotypes. Their CCA approach is equivalent to the multivariate analysis of variance or more generally Wilk's $\lambda$ test in a multivariate multiple linear regression (MMLR) approach [18]. According to O’Reilly et al. [13], MultiPhen and CCA performed very similarly in terms of power to detect causal variants except in case of low-frequency SNPs and non-normal phenotypes. Both O'Reilly et al. [13] and van der Sluis et al. [14] found significantly high power of CCA in case a subset of the traits was associated with the causal variant or gene. One major advantage of CCA is that it can easily be extended to incorporate multiple phenotypes as well as multiple SNPs (such as a gene). CCA provides the opportunity to study such a multivariate phenotype-genotype association analysis. But CCA cannot accommodate covariates, and one needs to perform the computationally intensive permutation testing to implement CCA in family data [17].

In this paper, we have used the equivalence between the CCA and the MMLR approach to analyze multiple traits as well as multiple SNPs in families. In fact, our ap- 
proach can be applied to samples involving both families as well as unrelated individuals. An advantage of the MMLR approach is that one can easily incorporate the covariates in the model. Another advantage of the MMLR approach is that it could provide a gene-based test of association without the need for permutation testing [16]. We propose here a rapid MMLR (RMMLR) approach in families. We have developed a related $R$ package to perform a multivariate gene-based GWAS with this RMMLR approach. We compared the performance of this RMMLR approach with several existing gene-based association analysis approaches, all of which require permutation or resampling techniques to estimate the null distribution of the test statistic in the presence of correlated SNPs or correlated phenotypes.

\section{Methods}

\section{Models}

For a set of pedigrees, each including one or more related individuals, let $y_{l i j}$ denote the measured phenotype $l$ on individual $j$ in pedigree $i\left(i=1, \ldots, m ; j=1, \ldots, n_{i} ; \sum_{i} n_{i}=n\right.$, and $\left.l=1, \ldots, L\right)$. Let $X_{i j k}$ denote the additive genotype score of the $k$-th $\operatorname{SNP}(k=1,2, \ldots, K)$ with alleles 'A' and 'a' of individual $j$ in pedigree $i$, where $X_{i j k}$ can take the values 0,1 , or 2 , depending on the number of minor alleles 'A' for individual $j$ in the $i$-th pedigree. Let vector $\boldsymbol{Y}_{l i}=\left\{y_{l i 1}, \ldots, y_{l i n_{i}}\right\}$ denote the vector of the $l$-th phenotype on individuals in the $i$-th pedigree, and let $\boldsymbol{X}_{i k}=\left\{X_{i 1 k}, \ldots, X_{i n_{k} k}\right\}$ denote the vector of genotypes of the $k$-th SNP from pedigree $i$. Let $C_{i}$ be a $n_{i} \times p$ matrix that contains the $p$ covariates of pedigree $i$. We are interested in methods to conduct a gene-based association analysis in pedigrees with one (unrelated individuals) or more related members with univariate or multivariate quantitative phenotypes.

For a quantitative phenotype $l$, we can do a single SNP association test for the $k$-th SNP, using the following linear mixed effect model,

$$
\begin{aligned}
\boldsymbol{Y}_{l i} & =\alpha_{l}+\boldsymbol{X}_{i k} \beta_{l k}+\boldsymbol{C}_{i} \boldsymbol{\beta}_{l c}+\boldsymbol{\varepsilon}_{l i} \\
& =\boldsymbol{X}_{i} \boldsymbol{\beta}_{l}+\boldsymbol{\varepsilon}_{l i}, i=1, \ldots, m,
\end{aligned}
$$

where $\alpha_{l}$ is the population mean, $\beta_{l k}$ is the additive fixed effect of the $k$-th SNP on the $l$-th trait, $\boldsymbol{\beta}_{l c}$ is a size $p$ vector of the covariate effects, and $\boldsymbol{\varepsilon}_{l i}$ is the random residual term for the $l$-th trait which is modeled as

$$
\boldsymbol{\varepsilon}_{l i} \stackrel{\text { ind }}{\sim} M V N\left(0, V_{l i}\right),
$$

assuming independent pedigrees. The $n_{i} \times n_{i}$ matrix $\boldsymbol{V}_{l i}$ is the variance-covariance matrix for the $l$-th trait. To simplify the notation, as shown on the right hand side of equ. 1, the observed data on the $(p+1)$ fixed predictors (1 SNP, $p$ covariates) and the intercept corresponding to pedigree $i$ are contained in the $n_{i} \times(p+2)$ design matrix $\boldsymbol{X}_{i}$, and the $(p+2)$ parameters are contained in the vector $\boldsymbol{\beta}_{l}$ for the $l$-th trait.

A maximum likelihood estimator of $\boldsymbol{V}_{l i}$ is calculated by $\hat{\boldsymbol{V}}_{l i}$ by assuming a multivariate normal distribution of the phenotype and a certain parametric structure of $\boldsymbol{V}_{l i}$ and considering the above model in equ. 2 but without the SNP term $\boldsymbol{X}_{i k} \beta_{l k}$. As shown by Li et al. [19], this approximation technique produces minimally biased estimates of $\boldsymbol{V}_{l i}$ under the assumption that a single SNP explains a small part of the total phenotypic variance. Let $\hat{V}_{l}$ be the block-diagonal matrix with $\hat{V}_{l i}$ s on its diagonal blocks, $i=1,2, \ldots$, $m$. We used bdspackage() in R [20] to compute the inverse of this block-diagonal matrix, denoted by $\hat{\boldsymbol{V}}_{l}^{-1}$ and a Cholesky decomposition of $\hat{\boldsymbol{V}}_{l}^{-1}$ as $\boldsymbol{V}_{l}^{-1}=S_{l} S_{l}^{T}$ [21-23]. The Cholesky decomposition of the positive definite covariance matrix $V_{l}^{-1}$ yields a matrix $S_{l}$ such that $V_{l}^{-1}=S_{l} S_{l}^{T}$, where $S_{l}$ is the lower triangular with positive diagonal elements. The matrix $S_{l}$ is called the 'Cholesky factor' of $V_{l}^{-1}$ and is equivalent to the square root of $V_{l}^{-1}$, denoted by $V_{l}^{-1 / 2}$. Hence $V_{l}^{-1 / 2} Y_{l}$ would multivariate normal distribution with mean $V_{l}^{-1 / 2}$ $\left(\boldsymbol{X} \boldsymbol{\beta}_{l}\right)$ and covariance matrix $V_{l}^{-1 / 2} V_{l} V_{l}^{-1 / 2}=\boldsymbol{I}$. In other words, the transformed data $V_{l}^{-1 / 2} \boldsymbol{Y}_{l}$ or $S_{l} \boldsymbol{Y}_{l}$ would produce observations following multivariate normal distribution with mean $S_{l} X \boldsymbol{\beta}_{l}$ and covariance matrix $\boldsymbol{I}$. Then a simple linear regression for these unrelated observations is carried out using the Cholesky factor-transformed data,

$$
Y_{l}^{*}=S_{l} \boldsymbol{Y}_{l}=S_{l} \boldsymbol{X} \boldsymbol{\beta}_{l}+\boldsymbol{\varepsilon}_{l}^{*},
$$

where $\boldsymbol{X}$ is the $n \times(p+2)$ matrix containing predictor values of all $m$ pedigrees, $\boldsymbol{Y}_{l}$ is the size $n$ vector of the phenotypes, $\boldsymbol{\beta}_{l}=\left(\alpha_{l}, \beta_{l k}\right.$, $\left.\boldsymbol{\beta}_{l c}\right)$ as defined in equ. 2 , and the residual term $\boldsymbol{\varepsilon}_{l}^{*}$ is distributed as $N(0, I)$. We make association calls based on the F test statistics from the simple linear regressions in equ. 4 . Here we want to test the null hypothesis $H_{0}: \beta_{l k}=0$, for $k=1,2, \ldots, K$ and $l=1,2, \ldots, L$.

\section{Combination Methods}

We used 2 combination methods to perform a gene-based association analysis. The combination methods combine the single SNP association $\mathrm{p}$ values to generate test statistics for the genebased tests. At the first stage, the p values for all SNPs within a gene are obtained through single SNP association tests based on standard regression models adjusting for other covariates. We used a rapid feasible generalized least square (RFGLS) approach [19] to perform the single SNP association analysis in pedigrees. The second stage requires the use of a single $\mathrm{p}$ value to represent the significance of each gene. We applied 2 methods, Fisher's combination test and the minP approach to calculate the significance of each gene.

minP Approach

Consider $K$ SNPs within a gene. The minP test statistic is given by

$$
p_{\text {min }}=\min _{i=1}^{K}\left\{K p_{i}\right\}
$$

for a single phenotype. We can extend it to consider testing association between genes and multiple phenotypes. For $L$ phenotypes and $K$ SNPs, the test statistic is given by

$$
p_{\min }=\min _{i=1}^{K L}\left\{K L p_{i}\right\} \text {. }
$$

Under the assumption of independence among the SNPs as well as among the phenotypes, this test statistic has a uniform distribution between $[0,1]$ under $H_{0}$. For positively correlated tests, this test tends to be conservative. There are alternative approaches, such as the Simes method or the Sidak method [24-26], that improve the conservativeness of the test. Otherwise, permutation procedures or parametric bootstrapping could be used to get valid $\mathrm{p}$ values. 
Fisher's Combination Test

The test statistic is given by

$$
\chi^{2}=-2 \sum_{i=1}^{K} \log p_{i}
$$

for $K$ SNPs and a single phenotype, which has a $\chi^{2}$ distribution with $2 K$ degrees of freedom under $H_{0}$, when $K$ tests are independent. We can extend this test for multiple phenotypes as well. The test statistic for testing no association between a gene and multiple phenotypes could be given by

$$
\chi^{2^{\prime}}=-2 \sum_{i=1}^{L K} \log p_{i}
$$

for $K$ SNPs and $L$ phenotypes. Assuming we have independent tests here, this test statistic has a $\chi^{2}$ distribution with $2 K L$ degrees of freedom under $H_{0}$. The major issue with this test is that it becomes anti-conservative for positively correlated tests, which could be the situation for SNPs in linkage disequilibrium (LD) or for positively correlated multiple traits. Hence a permutation procedure or parametric bootstrap technique (equivalent to the VEGAS-SUM approach) is needed if a valid $p$ value is to be obtained. The Versatile Gene-Based Test for Genome-Wide Association (VEGAS) [7] is a recent multivariate method that sums the association signal from all SNPs within a gene and corrects the sum for $\mathrm{LD}$ to generate a $\mathrm{p}$ value. One thing to note here is that VEGAS can only handle a single trait to perform a gene-based GWAS.

\section{Direct Modeling of Multiple SNPs and Multiple Phenotypes}

The combination methods provide a convenient way of proposing gene-based tests by utilizing the single SNP association $p$ values. However, by directly modeling multiple correlated traits and multiple SNPs, one could detect pleiotropic effects and could gain power by jointly modeling the effects of multiple SNPs in a single model.

\section{Multivariate Multiple Linear Regression}

Below we describe a MMLR model with $L$ traits and $K$ SNPs for $n$ unrelated individuals. Consider the following model for $n$ individuals with outcome variable $Y^{*}=\left(Y_{l}^{*}, l=1, \ldots, L\right)$ in equ. 4 of length $L$ :

$$
\boldsymbol{Y}_{l}^{*}=\boldsymbol{Z}_{i} \boldsymbol{\beta}+\boldsymbol{e}_{i} ; i=1, \ldots, n,
$$

where $e_{i}=\left(e_{i 1}, \ldots, e_{i L}\right)$ follows multivariate normal distribution, $\boldsymbol{Y}^{*}=\left(Y_{i 1}, \ldots, Y_{i L}\right)$ contains transformed data on $L$ phenotypes, $Z_{i}$ contains the transformed data $S_{l} \boldsymbol{X}_{i}$ on $K$ predictors (SNPs) and intercept for the $i$-th individual, and vector $\beta=\left(\beta_{0}, \beta_{1}, \beta_{2}, \ldots, \beta_{K}\right)$ represents the parameters corresponding to the intercept and $K$ SNPs. In this case, the within-sample sum of squares matrix $W$ is calculated as the covariance matrix of the residuals of the mod$\mathrm{el}$, and the between-sample sum of squares matrix is calculated as the covariance matrix of the fitted values $B$. We use Wilk's $\lambda$ test,

$$
\lambda=\frac{|W|}{|T|},
$$

where $T=W+B$ to test $H_{0}: \boldsymbol{\beta}=0$. It has been shown that for unrelated individuals, this MMLR approach is equivalent to the CCA approach mentioned before [18].

Compared with CCA, MMLR is much easier to expand to family structure and adjust for covariates. To apply it to family data, we used the computationally efficient RFGLS approach [19]. We calculated the within-cluster covariance matrix for each trait, and left-multiplied both the traits and the predictors separately by the inverse square root of the estimated covariance matrix. This transformation gave us the traits individually as independently distributed. We then computed the residuals for each trait from a model with intercept and modified covariates as well as the SNPs. Let us denote the residuals as $\boldsymbol{e}_{1}^{*}, \boldsymbol{e}_{2}^{*}, \ldots, \boldsymbol{e}_{L}^{*}$. We computed the covariance matrix of these residuals. This was our matrix $W$. In order to calculate $T$, we computed the residuals for each trait from a model with only intercept and the modified covariates. The covariance matrix of these residuals gave us $T$. We calculated our test statistic as

$$
\lambda=\left|\frac{W}{T}\right|,
$$

which was approximately distributed as $F$ with degrees of freedom $d f 1$ and $d f 2$, where

$$
d f 1=K L \text { and } d f 2=\left(n-3 / 2-\frac{K+L}{2}\right) s-\frac{K L}{2}+1 .
$$

We refer to this approach as RMMLR.

\section{Simulations}

To compare the different methods, we investigated their type I error and power by simulating data on nuclear families with 2 parents and 2 offspring. For simulating genotype data on a gene, we used a simulation setup very similar to that used by Li et al. [24]. First, we generated genotype data on 16 SNPs with different LD structures. We generated 3 independent blocks of SNPs: the first and the third block with 6 SNPs and the second block with 4 SNPs. For the low LD scenario, the pairwise LDs $\left(r^{2}\right)$ for the 3 blocks were $0.5,0.4$, and 0.5 ; while for the high LD scenario, the pairwise LDs $\left(r^{2}\right)$ were $0.9,0.8$, and 0.9 . The minor allele frequencies for the 3 blocks were $0.2,0.4$, and 0.25 . Note that $X_{i j k}$ denotes the genotype of the $k$-th SNP for the $j$-th member in the $i$-th family. For the $j$-th member in the $i$-th family, we drew a vector $(16 \times 1)$ of latent variables $U_{i j}$ from a multivariate normal with covariance matrix as (1) a low LD structure as shown in the appendix of Li et al. [24] or (2) a high LD structure as shown in the appendix of Li et al. [24]. Then by assuming HWE, we dichotomized $U_{i j k}$ such that if $U_{i j k}<p_{k}^{2}$, then $X_{i j k}=2$; if $U_{i j k}>1-\left(1-p_{k}\right)^{2}$, then $X_{i j k}=0$; otherwise $X_{i j k}=1, k=$ $1, \ldots, 16 . p_{k}$ is the minor allele frequency of the $k$-th SNP. We repeated this procedure to simulate genotypes of parents for all nuclear families. Then following Mendel's law, we generated genotypes of 2 offspring in each family. From each LD block of SNPs, the first SNP was selected as the causal SNP. We considered 2 different gene lengths such that the gene (1) contained the first 3 SNPs or (2) contained all 16 SNPs.

We then simulate 5 correlated traits. The simulation model is

$$
\boldsymbol{Y}_{l i}=\boldsymbol{G}_{i} \beta+\alpha_{l i}+e_{l i},
$$

where $Y_{l i}=\left(Y_{l i 1}, Y_{l i 2}, Y_{l i 3}, Y_{l i 4}\right)$ is the vector of the phenotypes for the $l$-th trait in the $i$-th family, parameter $\alpha_{l i}$ is the polygenic random effect for the $i$-th family and the $l$-th trait, and $\boldsymbol{G}_{i}$ is the matrix of the genotypes of the causal SNPs, which is a $4 \times r$ matrix if we have $r$ causal SNPs.

We allowed a correlation among different traits within each family. Suppose we have 5 traits, we then drew $\alpha_{i}=\left(\alpha_{1 i 1}, \alpha_{1 i 2}, \alpha_{1 i 3}\right.$, 
Table 1. Type I error for testing association between 5 traits and a 16-SNP gene: there is a moderate LD among the set of SNPs

\begin{tabular}{|c|c|c|c|c|c|c|c|c|c|}
\hline \multirow{2}{*}{$\begin{array}{l}\text { Trait } \\
\text { correlation }\end{array}$} & \multicolumn{3}{|c|}{$a=0.001$} & \multicolumn{3}{|c|}{$\alpha=0.0001$} & \multicolumn{3}{|c|}{$\alpha=0.00001$} \\
\hline & $\min \mathrm{P}$ & Fisher's test & RMMLR & $\min \mathrm{P}$ & Fisher's test & RMMLR & $\min \mathrm{P}$ & Fisher's test & RMMLR \\
\hline 0.2 & 0.0012 & 0.0071 & 0.00095 & $9.8 e^{-5}$ & 0.0018 & $9.75 e^{-5}$ & $9.4 \mathrm{e}^{-6}$ & $4.5 e^{-4}$ & $9.9 e^{-6}$ \\
\hline 0.4 & 0.0011 & 0.026 & 0.00095 & $9.5 e^{-5}$ & 0.0012 & $9.5 e^{-5}$ & $9.7 e^{-6}$ & $5.8 e^{-3}$ & $9.9 e^{-6}$ \\
\hline 0.6 & 0.0011 & 0.035 & 0.00096 & $9.8 \mathrm{e}^{-5}$ & 0.0017 & $9.5 e^{-5}$ & $9.6 \mathrm{e}^{-6}$ & $8.7 e^{-3}$ & $9.7 e^{-6}$ \\
\hline
\end{tabular}

$\left.\alpha_{1 i 4}, \ldots, \alpha_{5 i 1}, \alpha_{5 i 2}, \alpha_{5 i 3}, \alpha_{5 i 4}\right)$ from a multivariate normal with mean 0 and covariance matrix $\Sigma_{1}$ as:

$$
\left(\begin{array}{cccc}
\sigma_{a 1}^{2} 2 \Phi & \rho_{a b} \sigma_{a 1} \sigma_{a 2} * 2 \Phi & \ldots & \rho_{a b} \sigma_{a 1} \sigma_{a 5} 2 \Phi \\
\rho_{a b} \sigma_{a 1} \sigma_{a 2} * 2 \Phi & \sigma_{a 2}^{2} 2 \Phi & \ldots & \rho_{a b} \sigma_{a 2} \sigma_{a 5} 2 \Phi \\
& & \ldots & \\
\rho_{a b} \sigma_{a 1} \sigma_{a 5} 2 \Phi & \rho_{a b} \sigma_{a 2} \sigma_{a 5} 2 \Phi & \ldots & \sigma_{a 5}^{2} 2 \Phi
\end{array}\right),
$$

where $\Phi$ is the kinship matrix and $\rho_{a b}$ is the genetic correlation between traits, which is assumed in the simulation to be the same for all traits. In our simulation, for nuclear families with 2 offspring, we used the kinship matrix for offspring 1, offspring 2, parent 1 and parent 2 as:

$$
2 \Phi=\left(\begin{array}{cccc}
1 & 0.5 & 0.5 & 0.5 \\
0.5 & 1 & 0.5 & 0.5 \\
0.5 & 0.5 & 1 & 0 \\
0.5 & 0.5 & 0 & 1
\end{array}\right)
$$

and $\sigma_{a 1}^{2}=\sigma_{a 2}^{2}=\sigma_{a 3}^{2}=\sigma_{a 4}^{2}=\sigma_{a 5}^{2}=60$. We also simulated the environmental component $e_{i}=\left(e_{1 i 1}, e_{1 i 2}, e_{1 i 3}, e_{1 i 4}, \ldots . ., e_{5 i 1}, e_{5 i 2}, e_{5 i 3}, e_{5 i 4}\right)$ from a multivariate normal with mean 0 and covariance matrix as:

$$
\left(\begin{array}{cccc}
\sigma_{e 1}^{2} I & W & \ldots & W \\
W & \sigma_{e 2}^{2} I & \ldots & W \\
& & \ldots & \\
W & W & \ldots & \sigma_{e 5}^{2} I
\end{array}\right)
$$

where $\sigma_{e l}^{2}$ represents the environmental variance of trait $l, l=1, \ldots$, 5 . In the majority of the simulations, we considered no environmental correlation between the trait values for the same individual, i.e. $\boldsymbol{W}=\boldsymbol{I}$.

Additionally we assumed $\sigma_{e 1}^{2}=\sigma_{e 2}^{2}=\sigma_{e 3}^{2}=\sigma_{e 4}^{2}=\sigma_{e 5}^{2}=40$. For a 3-SNP gene, only the first block was used; while for the 16-SNP gene, all 3 blocks were used. We simulated 5 traits with different genetic correlations between the traits: $\rho_{a b}=0.33,0.67$, and 1 , which corresponds to a phenotypic correlation between the traits of 0.2 , 0.4 , and 0.6 , respectively. The first SNP of every block is the causal SNP under $H_{1}$, if the gene has covered that block. This means that the correlations between different traits are entirely coming from the genetic effect rather than the environmental effect.

Lastly, we add the causal SNP(s)' effect to the mean. The causal effect is obtained as:

$$
\beta_{k}=\sqrt{\frac{h_{k}^{2}}{2 p_{k}\left(1-p_{k}\right)}},
$$

Gene-Based GWAS with Multivariate Traits for the $k$-th causal SNP, where $h_{k}^{2}$ is the phenotypic variance explained by the $k$-th causal SNP. For $\rho_{a b}=0.33$ and 0.67 , we also performed 5,000 simulations to study the effect of an environmental correlation on power. We allowed for environmental correlation of $r=0.25$ and 0.5 between each pair of traits, i.e. we considered $\boldsymbol{W}=r \sigma_{e l} \sigma_{e k} \boldsymbol{I}$ for each pair of traits $l$ and $k$, where $l, k=1, \ldots, 5$.

\section{Results}

We first simulated data under $H_{0}$ to see the impact on the type I error. We simulated 100,000 datasets on 1,000 nuclear families under $H_{0}$ of no association between a gene and 5 traits. The type I error was estimated by calculating the proportion of times the asymptotic $\mathrm{p}$ value of the test statistic for each method was $\leq 0.001, \leq 0.0001$, and $\leq 0.00001$. We considered situations where the SNPs were in low LD and in high LD and present here only the results for the gene with 16 SNPs. The minP statistic in the low LD situation (table 1) was not that conservative, but it did get conservative in the high LD situation (table 2). On the other hand, Fisher's test was inflated due to the positive correlation among the traits as well as the SNPs. The type I error for Fisher's test was substantially higher for the high LD situation (table 2) as compared to the low LD condition (table 1) due to the higher positive correlation among the SNPs. Moreover, the type I error for Fisher's test was increased as the correlation among the traits increased from 0.2 to 0.6. These are known issues with the minP approach and Fisher's test, but we still wanted to demonstrate the extent of the impact of correlation among traits as well as SNPs on these 2 popular approaches, and the need to use approaches such as in Liu et al. [7] or in Li et al. [24] to correct for these issues with type I error. On the other hand, the RMMLR approach maintained a correct type I error under both the low LD and the high LD situation and for different degrees of correlation among the traits.

We next simulated data under $H_{1}$. We considered situations where the gene was associated with 1,2, 3, 4, or all 
Table 2. Type I error for testing association between 5 traits and a 16-SNP gene: there is a high LD among the set of SNPs

\begin{tabular}{|c|c|c|c|c|c|c|c|c|c|}
\hline \multirow{2}{*}{$\begin{array}{l}\text { Trait corre- } \\
\text { lation }\end{array}$} & \multicolumn{3}{|c|}{$a=0.001$} & \multicolumn{3}{|c|}{$\alpha=0.0001$} & \multicolumn{3}{|c|}{$\alpha=0.00001$} \\
\hline & $\min \mathrm{P}$ & Fisher's test & RMMLR & $\min \mathrm{P}$ & Fisher's test & RMMLR & $\min \mathrm{P}$ & Fisher's test & RMMLR \\
\hline 0.2 & 0.0007 & 0.026 & 0.0011 & $8.03 e^{-5}$ & 0.0016 & $9.97 e^{-5}$ & 0.000 & $5.5 e^{-3}$ & $1.004 \mathrm{e}^{-5}$ \\
\hline 0.4 & 0.0008 & 0.048 & 0.00095 & $5.1 e^{-5}$ & 0.026 & $1.02 \mathrm{e}^{-4}$ & 0.000 & 0.0015 & $1 e^{-5}$ \\
\hline 0.6 & 0.0008 & 0.076 & 0.001 & 0.000 & 0.048 & $9.5 e^{-5}$ & 0.000 & 0.031 & $9.9 \mathrm{e}^{-6}$ \\
\hline
\end{tabular}

Table 3. Power of the different methods for the 3-SNP gene (1 causal SNP): there is a moderate LD

\begin{tabular}{|c|c|c|c|c|c|c|}
\hline \multirow{2}{*}{$\begin{array}{l}\text { Trait } \\
\text { correla- } \\
\text { tion }\end{array}$} & \multicolumn{3}{|c|}{ All 5 traits associated } & \multicolumn{3}{|c|}{3 traits associated } \\
\hline & $\begin{array}{l}\min \mathrm{P} \\
\text { (empirical) }\end{array}$ & $\begin{array}{l}\text { Fisher's test } \\
\text { (empirical) }\end{array}$ & $\begin{array}{l}\text { RMMLR } \\
\text { (asymptotic) }\end{array}$ & $\begin{array}{l}\min \mathrm{P} \\
\text { (empirical) }\end{array}$ & $\begin{array}{l}\text { Fisher's test } \\
\text { (empirical) }\end{array}$ & $\begin{array}{l}\text { RMMLR } \\
\text { (asymptotic) }\end{array}$ \\
\hline 0.2 & 0.687 & 0.815 & 0.653 & 0.574 & 0.425 & 0.596 \\
\hline 0.4 & 0.596 & 0.535 & 0.463 & 0.536 & 0.247 & 0.639 \\
\hline 0.6 & 0.476 & 0.475 & 0.285 & 0.406 & 0.149 & 0.801 \\
\hline
\end{tabular}

5 traits. For each scenario, we simulated 5,000 datasets on 500 nuclear families. We also considered 2 different gene lengths and different LD patterns among the SNPs to see the impact of gene length and LD on power. We report the empirical power for the minP and Fisher's test in order to correct for any inflatedness or conservativeness in the type I error. The empirical power was calculated by counting the proportion of times the trait SNP was called significant ( $\mathrm{p}$ value $\leq$ the empirical 0.001-th cutoff) out of the 5,000 simulations. Since the RMMLR approach maintained a correct type I error rate, we report the asymptotic power for the approach.

We first simulated data on 500 nuclear families for a 3 -SNP gene with 1 causal SNP explaining $0.6 \%$ of the phenotype variance. Table 3 shows the empirical power of the minP and Fisher's test and the asymptotic power of the RMMLR approach at a type I error level of 0.001 . We report here the situations where the causal SNP is associated with all traits and the situation where 3 of the traits are associated with the SNP. In case of complete association, the minP and Fisher's test had comparable power (table 3) for different intertrait correlations. The power of the RMMLR approach was relatively lower than that of the other 2 approaches. All of them lost power as we increased the intertrait correlation among individuals. On the other hand, the RMMLR approach had significantly higher power than the other 2 approaches in case a subset of the traits was associated with the gene (table 3). Moreover, the power for gene detection generally went up for the RMMLR approach when the trait correlation was increased in case the gene was associated with a subset of the traits, but the other 2 approaches lost power as the trait correlation went higher. Especially, Fisher's test performed very poorly when the trait correlation was high and a subset of the traits was associated with the gene.

We next considered a 16-SNP gene with 3 causal SNPs explaining a total of $0.9 \%$ variability for each of the associated traits and where the gene was associated with 1,2 , 3,4 , or all 5 traits. Figure 1 shows the performance of the different approaches when the correlation among the traits was $0.2,0.4$, and 0.6. The SNPs were in moderate LD. In general, the RMMLR approach had significantly better or equivalent power to the other 2 approaches except for the situation when the gene was associated with all traits. It also appeared to perform better when the residual trait correlation was moderate or high (0.4 or 0.6$)$. Especially when the trait correlation was 0.6 , the power was substantially higher in case the gene was associated with a subset of the traits (fig. 1). On the other hand, the power for both the minP and Fisher's test decreased as the trait correlation was increased. Fisher's test gained little power for the 16-SNP gene compared to the 3-SNP gene situation (table 3) due to the fact that multiple SNPs were associated with the traits. On the other hand, the minP lost significant power for the 16-SNP gene (fig. 1) as compared to the 3-SNP gene study due to not accounting for multi-SNP association. As expected, Fisher's test had very good power when most or all traits were associated with the gene, but its inflated type I error due to a high correlation among the traits or SNPs significantly limited its usefulness to perform a gene-based association analysis.

We also checked the behavior of these approaches for a 16-SNP gene when the LD among the SNPs was high and the gene was associated with 3 out of the 5 traits. After correcting for the type I error in the minP and Fisher's test, all approaches maintained a similar power for both 


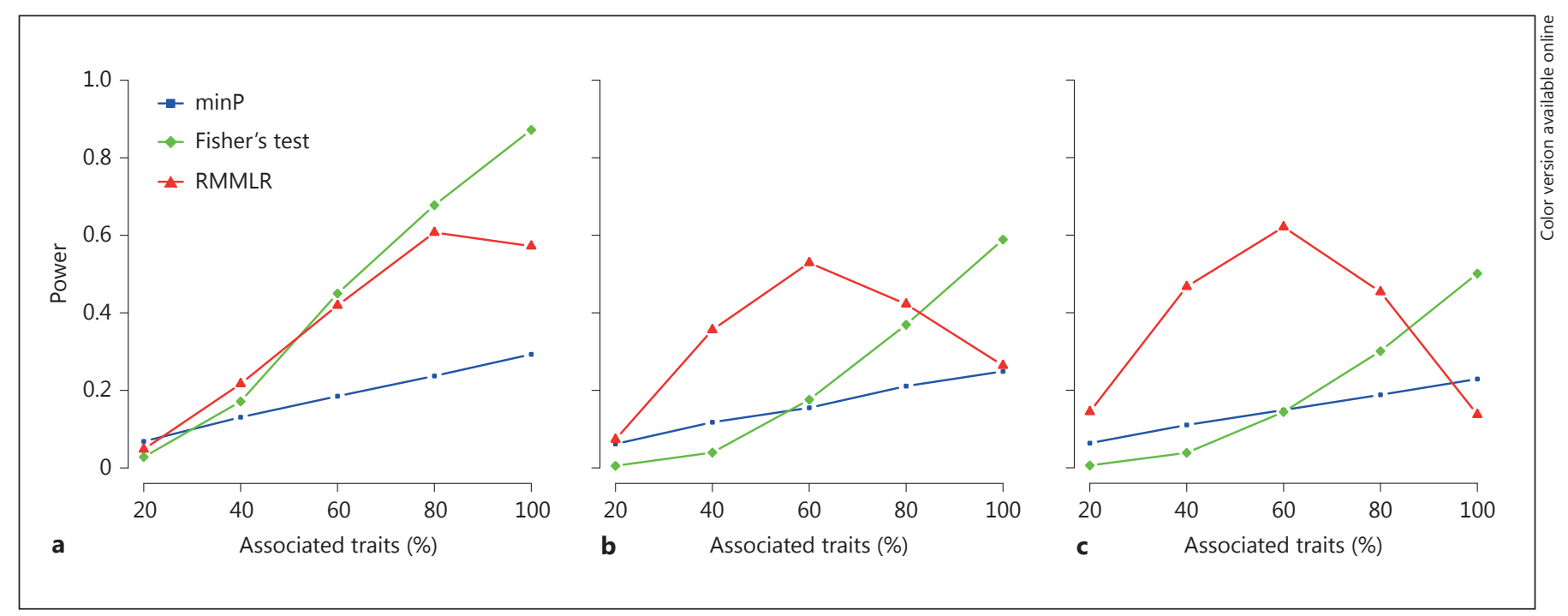

Fig. 1. Performance of the multivariate tests of association. The empirical powers for the minP, Fisher's test, and asymptotic power for the RMMLR approach are shown for different numbers of traits being associated with a 16-SNP gene. The residual trait correlation was $0.2(\mathbf{a}), 0.4$ (b), and 0.6 (c).

Table 4. Power of the different methods for the 16-SNP gene (3 causal SNPs): LD is high and 3 of these 5 traits are associated with the causal SNPs

\begin{tabular}{llll}
\hline Trait correlation & $\begin{array}{l}\text { minP } \\
\text { (empirical) }\end{array}$ & $\begin{array}{l}\text { Fisher's test } \\
\text { (empirical) }\end{array}$ & $\begin{array}{l}\text { RMMLR } \\
\text { (asymptotic) }\end{array}$ \\
\hline 0.2 & 0.171 & 0.450 & 0.419 \\
0.4 & 0.170 & 0.250 & 0.523 \\
0.6 & 0.152 & 0.144 & 0.675 \\
\hline
\end{tabular}

the low LD (table 4) and the high LD situation (fig. 1). We also studied the effect of increasing environmental correlation for this 16-SNP gene with 3 causal SNPs, when the LD among the SNPs was high and the gene was associated with 3 out of the 5 traits. For a genetic correlation of $\rho_{a b}=0.33$ and 0.67 , we considered an environmental correlation $(r)$ of 0.25 and 0.5 . The overall residual trait correlation was 0.3 and 0.4 for $\rho_{a b}=0.33$ and $r=0.25$ and 0.5 , respectively. The overall residual trait correlation was 0.5 and 0.6 for $\rho_{a b}=0.67$ and $r=0.25$ and 0.5 , respectively. Our findings were very similar to those in table 4 . The power substantially went up for the RMMLR approach with the increase in residual trait correlation, but both the $\operatorname{minP}$ and Fisher's test lost power with the increase in residual trait correlation (results not shown).

In summary, the RMMLR approach performed really well when some but not all correlated traits were associ- ated with the gene. Moreover, the RMMLR approach maintained the correct type I error even for highly correlated traits and for markers in high LD. Hence this RMMLR approach provides a computationally efficient technique for gene-based GWAS using unrelated individuals or families. Under complete association, Fisher's test performed really well detecting association, but we had to correct for an inflated type I error by simulating data under $H_{0}$. The minP approach performed well for the 3-SNP gene, but its power was substantially lower than that of the other 2 approaches for the 16-SNP gene.

\section{Real Data Analysis}

A genome-wide gene association analysis was carried out using our proposed RMMLR approach and other existing tests on data from the Minnesota Center for Twin and Family Research (MCTFR), which consists of an interrelated series of longitudinal twin, family, and adoption studies. The MCTFR sample and genotyping methods have been described by Miller et al. [27]. The MCTFR phenotypes have been described by Hicks et al. [28]. An initial SNP-based GWAS analysis has been reported by McGue et al. [29]. Briefly, the sample used here includes 7,188 individuals of European ancestry clustered in 2,300 nuclear families. Included are 2,072 mothers (mean age \pm SD at assessment: $42.8 \pm 5.3$ years), 1,780 fathers (44.9 \pm 5.7 years), 1,788 daughters (18.0 \pm 0.8 years), and 1,548 sons (17.9 \pm 0.7 years). Families are distinguished by the 


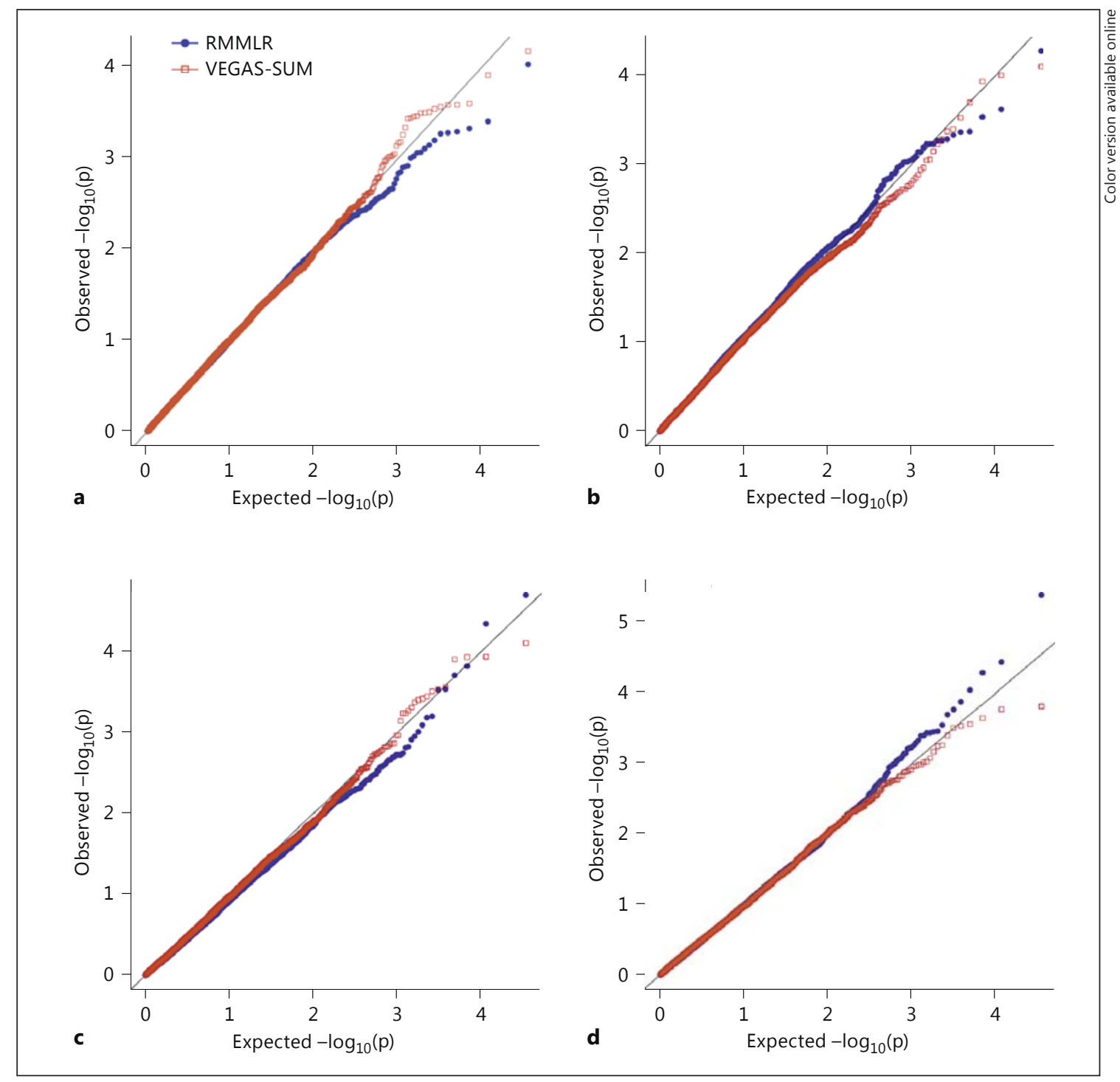

Fig. 2. $-\log _{10}$ (p values) comparing the VEGAS-SUM and the RMMLR approach for each of the 4 phenotypes: NIC FAC (a), DRG FAC (b), ALC FAC (c), BD FAC (d). There were 17,600 genes.

relationship of the 2 participating offspring, who were either monozygotic twins, same-sex dizygotic twins, full biological siblings, adoptive siblings, or mixed adopted/ biological siblings. The sample was genotyped using Illumina's Human 660W-Quad Array (Illumina, Inc., San Diego, Calif., USA) and a description of the quality control procedures is given in Miller et al. [27]. Of the 561,490 SNP markers on the array, 527,829 $(94.0 \%)$ passed all quality control filters. The quality control filters on the samples resulted in a removal of $<2 \%$ of the genotyped participants. 'European ancestry' was based on self-report and was confirmed through the principal compo- nent analysis of the covariance matrix of marker data as implemented in the Eigensoft package [30].

The 4 quantitative clinical phenotypes used in this analysis are all indicators of a higher-order construct known as externalizing or disinhibitory psychopathology [31]. These phenotypes were derived using a higher-order factor analytic approach [28] and included: (1) a nicotine factor (NIC FAC), a measure of symptoms of nicotine dependence; (2) an alcohol consumption factor (ALC FAC), a measure of quantity and frequency indicators of alcohol consumption; (3) an illicit drug consumption factor (DRG FAC), a measure of the use and symptoms of abuse and 
dependence on illicit substances, and (4) a behavioral disinhibition factor (BD FAC), a measure of indicators of nonsubstance use of behavioral disinhibition. In the MCTFR, parents are typically assessed a single time, while offspring are assessed longitudinally, spanning adolescence through early adulthood. The factor scores here for the offspring are based on the assessment completed nearest their 17th birthday so long as that assessment was completed when they were between 16.5 and 21 years. The average (range) correlation among these 4 phenotypes was $0.56(0.46-0.68)$. We conducted both univariate and multivariate gene-based GWAS scans for these 4 phenotypes using our RMMLR approach. Covariates in these analyses included sex, age, generation (i.e. parent vs. offspring) and the first 10 principal components of the genotype covariance matrix derived by the Eigensoft package [30]. For a comparison, we also report the findings from the VEGAS-SUM gene-based GWAS. One important issue to note is that the RMMLR approach is much more computationally efficient than the VEGAS-SUM approach. Moreover, the RMMLR approach can perform a multivariate gene-based GWAS, which could have more power to detect multi-SNP association within a gene.

We first used our RFGLS approach [19] to perform single SNP association tests. The stepparents in the sample were treated as unrelated individuals and the rest of the sample was classified into different family types. We assumed a 12-parameter structure for the residual covariance matrix to account for the difference in variances and correlation among the pedigree members in different family types. We then assumed a normal distribution of the traits to estimate the covariance matrix through a maximum likelihood estimation. The VEGAS-SUM approach was then used to perform a gene-based GWAS using the single SNP association test statistics. Next we used the same gene definition as used by VEGAS to implement the RMMLR approach. We estimated the pairwise correlations among the SNPs within a gene and removed all except one of the SNPs in perfect LD with each other for our gene-based analysis.

The VEGAS-SUM approach is similar to Fisher's test we used in our simulation study. The positive correlation among the SNPs within a gene can cause an inflated VEGAS test statistic. The bootstrapping in the VEGAS-SUM approach adjusts for such an inflatedness. We performed a maximum of $10^{7}$ such bootstrap iterations to estimate the null distribution of the VEGAS test statistic. Of note, it took us $6.02 \mathrm{~h}$ of CPU time to analyze the 17,600 genes using 520,478 SNPs, and it used up to 20 GB RAM to conduct this gene-based GWAS. On the other hand, the

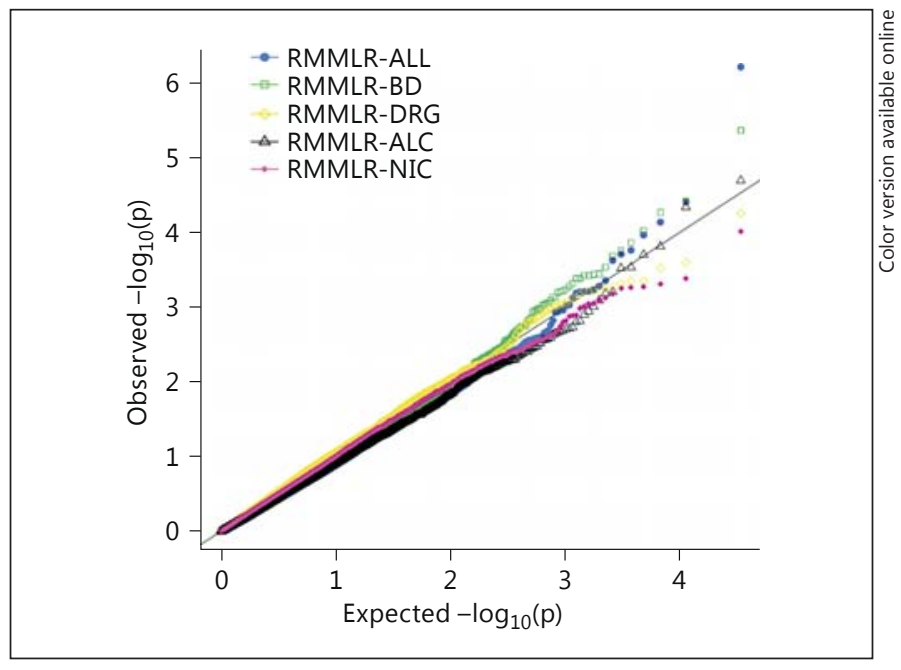

Fig. 3. $-\log _{10}$ ( $p$ values) using the RMMLR approach for each of the 4 phenotypes (NIC FAC, ALC FAC, DRG FAC, and BD FAC) as well as for the 4 phenotypes analyzed together (ALL).

RMMLR approach was computationally very efficient and took significantly less time ( $2.35 \mathrm{~h}$ of CPU time) and memory (approximately 1.95 GB RAM) to complete the analysis. Moreover, we were able to perform both univariate and multivariate gene-based GWAS with this approach (fig. 2, 3). The multi-trait multi-SNP association analysis produced several more significant findings as compared to the individual univariate multi-SNP association analysis, supporting our hypothesis of a possible association between a subset of phenotypes and genes (fig. 3). Through our multivariate analysis, we found the gene RPL7 on chromosome 8q21.11 to be genome-wide significant $\left(\mathrm{p}=6.135530 \times 10^{-7}\right)$ even after applying Bonferroni's correction for multiple testing $(0.05 / 17,600=$ $\left.2.84 \times 10^{-6}\right)$. The corresponding univariate $\mathrm{p}$ values produced by the RMMLR approach were 0.007322907 for BD FAC, $2.023403 \times 10^{-5}$ for ALC FAC, 0.001710981 for DRG FAC, and 0.004259516 for NIC FAC. None of them were significant at the genome-wide level. Other than RPL7, we found gene MYL1 on chromosome 2q33-q34 and gene $\mathrm{RDH} 10$ on chromosome 8q21.11 to have pvalues $<0.00001$ in the multivariate gene-based association analysis.

\section{Discussion}

Very few methods are available to model association between multiple traits and multiple SNPs. In the study of a complex disease, several correlated traits are often 
measured as risk factors for the disease. Analyzing multiple disease-related phenotypes could potentially increase power to detect association of SNPs/genes with a disease. Moreover, this joint analysis could reveal some pleiotropic genes involved in the biological development of the disease. Our flexible RMMLR approach could provide a gene-based association analysis with univariate as well as multivariate traits. Our approach could be applied to secondary continuous traits collected as part of a casecontrol study using the retrospective likelihood method developed for mapping secondary phenotypes using regression models (SPREG) in case-control studies [32]. One could also use this approach for interaction detection by adding higher-order interaction terms in the regression model, if there is any prior evidence or interest in modeling interaction between certain SNPs in a gene. The RMMLR approach is computationally efficient and simulation studies showed a substantial power gain when the gene is associated with a subset of the traits, especially in the presence of a moderate or high residual trait correlation. The method remains valid even when there is a high LD among the SNPs, and hence the asymptotic Wilk's $\lambda$ test can be used to assess the association. We have developed an $\mathrm{R}$ package to perform gene-based GWAS analyses with this RMMLR approach.

The CCA or MMLR approach has already been successfully implemented for unrelated individuals. Tang and Ferreira [16] demonstrated the advantage of the CCA approach in a multi-trait and multi-SNP association analysis. Many GWAS involve family samples. The technique suggested by Ferreira and Purcell [17] for family data is computationally intensive and can only be implemented for a single SNP association analysis. Here we propose a technique that can be implemented in families as well as in unrelated individuals. It is computationally efficient and captures the desirable features of the CCA algorithm. It has much higher power compared to the existing approaches, when a gene is associated with only a subset of the traits.

It could get computationally intensive to compute the variance-covariance matrix in the MMLR approach (equ. 4) for every gene in a gene-based GWAS analysis. Our RMMLR approach is an approximation technique that avoids such intensive computation. The method works based on the principle that most of the genes in a GWAS study have very little or no effect on the traits. Hence estimating the variance matrix without including the gene effect does not cause much loss in power. On the other hand, if a gene is associated with the traits, our approximation technique can detect it. In our simulation studies, the RMMLR approach had $>80 \%$ power to detect a gene explaining $0.6 \%$ of the phenotypic variance, in case the gene is associated with a subset of the traits, with a sample size of 500 families, and with a familial correlation of 0.6 .

Our univariate gene-based association testing with VEGAS was computationally intensive; it took us $6.02 \mathrm{~h}$ of CPU time to test 17,600 genes using 520,478 SNPs, and it used up to 20 GB RAM to conduct this gene-based GWAS. On the other hand, the RMMLR approach was easy to implement, and we were able to use the asymptotic $\mathrm{p}$ values of the gene-based test statistic to assess the significance of the genes.

One disadvantage of the RMMLR approach over the combination methods is that it requires the availability of genotype and phenotype data on individuals in order to perform the multivariate analysis. On the other hand, the combination methods require only the $\mathrm{p}$ values from a single SNP association analysis of univariate traits and hence might be more preferable to the user in some situations. We also need to remove SNPs in complete LD from the regression model to control for perfect multicollinearity for our RMMLR approach. Another issue is that the RMMLR approach assumes multivariate normality of the trait. Ferreira and Purcell [17] found that the CCA approach remains valid even for non-normal traits. O'Reilly et al. [13] found that in unrelated samples, the CCA approach was sensitive to non-normality for low-frequency SNPs. We expect a similar behavior of the RMMLR approach in the presence of non-normality, but we intend to investigate it extensively through simulation studies in the future.

In conclusion, our proposed RMMLR approach provides a useful framework for the detection of gene-based association with single or multivariate traits, which could outperform the commonly used permutation-based approaches, especially in case the gene is associated with a subset of the traits. Our gene-based association analysis with multiple traits from the MCTFR dataset had some improved $\mathrm{p}$ values as compared to the univariate RMMLR tests, indicating evidence of an association between the gene and a subset of the multiple correlated traits.

\section{Acknowledgements}

This research was supported in part by NIH grant U01DA024417, NIH grant R21-DK089351, and NIH grant R01DA033958. The authors would like to thank the 2 anonymous reviewers for their helpful comments and suggestions. 


\section{References}

$>1$ Visscher PM, Brown MA, McCarthy MI, Yang J: Five years of GWAS discovery. Am J Hum Genet 2012;90:7-24.

-2 Roeder K, Bacanu SA, Sonpar V, Zhang X, Devlin B: Analysis of single-locus tests to detect gene/disease associations. Genet Epidemiol 2005;28:207-219.

-3 Lesnick TG, Papapetropoulos S, Mash DC, Ffrench-Mullen J, Shehadeh L, et al: A genomic pathway approach to a complex disease: axon guidance and Parkinson disease. PLoS Genet 2007;3:e98.

$>4$ Ballard DH, Cho J, Zhao H: Comparisons of multi-marker association methods to detect association between a candidate region and disease. Genet Epidemiol 2010;34:201-212.

$\checkmark 5$ Neale BM, Sham PC: The future of association studies: gene-based analysis and replication. Am J Hum Genet 2004;75:353-362.

6 Wang K, Li M, Bucan M: Pathway-based approaches for analysis of genomewide association studies. Am J Hum Genet 2007;81:12781283.

7 Liu JZ, McRae AF, Nyholt DR, Medland SE, Wray NR, et al: A versatile gene-based test for genome-wide association studies. Am J Hum Genet 2010;87:139-145.

$>8$ Purcell S, Neale B, Todd-Brown K, Thomas L, Ferreira MA, Bender D, Maller J, Sklar P, de Bakker PI, Daly MJ, Sham PC: PLINK: a tool set for whole-genome association and population-based linkage analyses. Am J Hum Genet 2007;81:559-575.

9 Germain M, Saut N, Greliche N, Dina C, Lambert JC, Perret C, et al: Genetics of venous thrombosis: insights from a new genome wide association study. PLoS One 2011;6:e25581.

10 Souto JC, Almasy L, Borrell M, Blanco-Vaca F, Mateo J, et al: Genetic susceptibility to thrombosis and its relationship to physiological risk factors: the GAIT study. Genetic Analysis of Idiopathic Thrombophilia. Am J Hum Genet 2000;67:1452-1459.
11 Klei L, Luca D, Devlin B, Roeder K: Pleiotropy and principal components of heritability combine to increase power for association analysis. Genet Epidemiol 2008;32:9-19.

12 Lange C, van Steen K, Andrew T, Lyon H, DeMeo DL, et al: A family-based association test for repeatedly measured quantitative traits adjusting for unknown environmental and/or polygenic effects. Stat Appl Genet Mol Biol 2004;3:Article17.

13 O'Reilly PF, Hoggart CJ, Pomyen Y, Calboli FCF, Elliott P, et al: MultiPhen: joint model of multiple phenotypes can increase discovery in GWAS. PLoS One 2012; 7:e34861.

14 van der Sluis S, Posthuma D, Dolan CV: TATES: efficient multivariate genotype-phenotype analysis for genome-wide association studies. PLoS Genet 2013;9:e1003235.

15 Maity A, Sullivan PF, Tzeng JY: Multivariate phenotype association analysis by marker-set kernel machine regression. Genet Epidemiol 2012;36:686-695.

16 Tang S, Ferreira MAR: A gene-based test of association using canonical correlation analysis. Bioinformatics 2012;28:845-850.

17 Ferreira MAR, Purcell SM: A multivariate test of association. Bioinformatics 2009;25:132133.

18 Muller KE, Peterson BL: Practical methods for computing power in testing the multivariate general linear hypothesis. Comput Stat Data Anal 1984;2:143-158.

-19 Li X, Basu S, Miller MB, Iacono WG, McGue $\mathrm{M}$ : A rapid generalized least squares model for a genome-wide quantitative trait association analysis in families. Hum Hered 2011;71: 67-82.

20 Hornik K: The R FAQ (ISBN 3-900051-08-9, 2012). http://CRAN.R-project.org/doc/FAQ/ R-FAQ.html.

21 Golub GH, Van Loan CF: Matrix Computations. Baltimore, The Johns Hopkins University Press, 1996.

22 Horn RA, Johnson CR: Matrix Analysis. Cambridge, Cambridge University Press, 1985.
23 Trefethen LN, Bau D: Numerical Linear Algebra. Philadelphia, Society for Industrial and Applied Mathematics (SIAM), 1997.

24 Li MX, Gui HS, Kwan JS, Sham PC: GATES: a rapid and powerful gene-based association test using extended Simes procedure. Am J Hum Genet 2011;88:283-293.

25 Sidak Z: Rectangular confidence regions for the means of multivariate normal distributions. J Am Stat Assoc 1967;62:626-633.

26 Simes RJ: An improved Bonferroni procedure for multiple tests of significance. Biometrika 1986;73:751-754.

-27 Miller MB, Basu S, Cunningham JM, Eskin E, Malone SM, et al: The Minnesota Center for Twin and Family Research genome-wide association study. Twin Res Hum Genet 2012; 15:767-774.

28 Hicks BM, Schalet BD, Malone SM, Iacono WG, McGue M: Psychometric and genetic architecture of substance use disorder and behavioral disinhibition measures for genetic association studies. Behav Genet 2011;41: 459-475.

29 McGue M, Zhang Y, Miller MB, Basu S, Vrieze $S$, et al: A genome-wide association study of behavioral disinhibition. Behav Genet 2013;43:363-373.

30 Price L, Patterson NJ, Plenge RM, Weinblatt ME, Shadick NA, Reich D: Principal components analysis corrects for stratification in genome-wide association studies. Nat Genet 2006;38:904-909.

31 Iacono WG, Malone SM, McGue M: Behavioral disinhibition and the development of early-onset addiction: common and specific influences. Annu Rev Clin Psychol 2008;4: 325-348.

32 Lin DY, Zeng D: Proper analysis of secondary phenotype data in case-control association studies. Genet Epidemiol 2009;33:256-265. 